ARTICLE

Received 3 Jul 2012 | Accepted 3 Oct 2012 | Published 13 Nov $2012 \quad$ DOl: 10.1038/ncomms2185

\title{
Tracking lithium transport and electrochemical reactions in nanoparticles
}

Feng Wang ${ }^{1}$, Hui-Chia $\mathrm{Yu}^{2}$, Min-Hua Chen ${ }^{2}$, Lijun $\mathrm{Wu}^{1}$, Nathalie Pereira ${ }^{3}$, Katsuyo Thornton ${ }^{2}$, Anton Van der Ven², Yimei Zhu', Glenn G. Amatucci ${ }^{3} \&$ Jason Graetz ${ }^{1}$

Expectations for the next generation of lithium batteries include greater energy and power densities along with a substantial increase in both calendar and cycle life. Developing new materials to meet these goals requires a better understanding of how electrodes function by tracking physical and chemical changes of active components in a working electrode. Here we develop a new, simple in-situ electrochemical cell for the transmission electron microscope and use it to track lithium transport and conversion in $\mathrm{FeF}_{2}$ nanoparticles by nanoscale imaging, diffraction and spectroscopy. In this system, lithium conversion is initiated at the surface, sweeping rapidly across the $\mathrm{FeF}_{2}$ particles, followed by a gradual phase transformation in the bulk, resulting in 1-3 nm iron crystallites mixed with amorphous LiF. The real-time imaging reveals a surprisingly fast conversion process in individual particles (complete in a few minutes), with a morphological evolution resembling spinodal decomposition. This work provides new insights into the inter- and intra-particle lithium transport and kinetics of lithium conversion reactions, and may help to pave the way to develop highenergy conversion electrodes for lithium-ion batteries.

\footnotetext{
${ }^{1}$ Brookhaven National Laboratory, Upton, New York 11973, USA. ${ }^{2}$ Department of Materials Science and Engineering, University of Michigan, Ann Arbor, Michigan 48109, USA. ${ }^{3}$ Department of Materials Science and Engineering, Rutgers University, North Brunswick, New Jersey 08902, USA. Correspondence and requests for materials should be addressed to F.W. (email: fwang@bnl.gov) or to J.G. (email: graetz@bnl.gov).
} 
ithium-ion batteries are the preferred energy storage devices for portable electronics and will have an important role in large-scale applications, such as electric vehicles and gridscale storage ${ }^{1}$. The transition from portable electronics, which typically last 2-3 years, to vehicles and grid, with expected lifetime greater than 10 years, will require substantial improvements in calendar and cycling life. In addition, automotive applications require at least a two-fold improvement in energy and power densities. One of the promising classes of electrode materials that could meet these requirements is lithium conversion compounds, which have the advantage of accommodating more than one lithium per transition metal, boasting high theoretical capacities ${ }^{2-4}$, and in some cases, exhibit excellent capacity retention. A recent study of lithium conversion in the $\mathrm{FeF}_{2}$ cathode offered the first experimental evidence of the formation of a conductive iron network, ${ }^{5}$ which may provide the pathway for electron transport necessary for reversible lithium cycling ${ }^{2,4,6,7}$. However, these electrodes are typically plagued by poor cycling rate and a large cycling hysteresis, 8 . Improvements will require a better understanding of the phase nucleation and evolution, electron and ion transport, and reaction kinetics, which are difficult to determine by ex-situ measurements.

Most of the available in-situ techniques, such as those based on hard X-ray scattering, are suited for studying bulk electrodes and have inadequate spatial resolution for exploring nanoscale morphological and structural changes, and determining where and how new phases nucleate and propagate ${ }^{10,11}$. Transmission electron microscopy (TEM), capable of exceptional spatial resolution has, until recently, been unsuitable for these studies due to issues associated with the limited space between the objective lens pole pieces, low electron transparency and an incompatibility of the liquid electrolyte with the high vacuum environment. A few recent attempts to circumvent these issues involved sealed liquid cells or open cells with an ionic liquid (low vapour pressure) or solid electrolyte ${ }^{12-16}$. However, these approaches are often unsuitable for high-resolution studies of nanoparticles because of reduced electron transparency (as a result of the thick window) and/or the requirement for special electrode morphologies (for example, nanowires/rods, or thin films). Importantly, these techniques are mostly incapable of tracking the structural and chemical evolution occurring in the electrodes during charge/discharge.

Here we develop a new, simple in-situ electrochemical cell for the TEM by adapting a commercially available biasing system, as illustrated in Fig. 1a. A lithium metal counter electrode was applied to a sharp tungsten tip that was attached to a piezo-driven biasing-probe built into the TEM-scanning tunnelling microscopy sample stage (Nanofactory Instruments $\mathrm{AB}$ ). The lithium electrochemical reaction with $\mathrm{FeF}_{2}$, which occurs via a conversion process $\left(\mathrm{FeF}_{2}+2 \mathrm{Li}^{+}+2 \mathrm{e}^{-} \rightarrow 2 \mathrm{LiF}+\mathrm{Fe}\right)$, was investigated using $\mathrm{FeF}_{2}-\mathrm{C}$ nanocomposite electrodes, with $\mathrm{FeF}_{2}$ nanoparticles supported on carbon. The evolution of the electrode undergoing the conversion reaction was captured over a range of length scales-capturing changes in the composite (micron-scale) and within an individual particle (sub-nanometre scale), via real-time TEM imaging, electron diffraction (ED) and electron energy-loss spectroscopy (EELS) recorded at sub-second temporal resolution. First-principles calculations and phase-field simulations were employed to elucidate the origin of the observed morphological and phase evolution, and the underlying mechanisms involved in the electrochemical conversion reaction. In this study, direct experimental observation, supported by computation, reveals that the lithium conversion is initiated at the surface and then propagates into the bulk with a morphological evolution resembling spinodal decomposition, resulting in the formation of a network of interconnected $\mathrm{Fe}$ nanoparticles $(1-3 \mathrm{~nm})$ within the amorphous $\mathrm{LiF}$.

\section{Results}

Lithium reaction with individual nanoparticles. The $\mathrm{FeF}_{2}-\mathrm{C}$ composite electrodes were made with as-synthesized $10-20 \mathrm{~nm}$ $\mathrm{FeF}_{2}$ particles dispersed on thin carbon films and further incorporated into the setup illustrated in Fig. 1a for the in-situ TEM measurements. The real-time morphological changes occurring during lithiation were recorded in Supplementary Movies 1 and 2, and annular dark-field (ADF) images (Fig. 1b, c). The reaction front propagates extremely fast and sweeps across the collection of particles in region $I$ within a few seconds (Supplementary Movie 1). In contrast, the reaction is delayed (by about $10 \mathrm{~min}$ ) in region $I I$, and does not occur at all in region $I I I$, even after more than $20 \mathrm{~min}$. The reduced activity in these regions is likely due to poor contact between particles and the current collector, which inhibits ion and electron transport. On the other hand, this observation confirms that the observed morphological changes are due to the conversion reaction rather than beam-induced phase decomposition that may also occur under intense electron irradiation $^{17}$.

The electrochemical conversion of a single particle is shown in the time-lapse images in Fig. 1c, revealing a rapid surface reaction leading to the formation of small, sub-nanometre Fe particles, followed by the reaction in the bulk, resulting in slightly larger (1-3 nm) Fe particles. The lithiation under an external bias clearly indicates two different timescales for inter- and intra-particle $\mathrm{Li}^{+}$ transport (see also Supplementary Movie 2 and Supplementary Fig. S1). The Li ions appear to move quickly via surface diffusion along the carbon film and $\mathrm{FeF}_{2}$ particles, and then penetrate into the bulk at a much slower rate.

There is no observable coarsening of the newly formed $\mathrm{Fe}$ nanoparticles throughout the conversion period (Fig. 1b,c and Supplementary Movie 1). This observation suggests an immediate decomposition reaction that occurs locally, and that the LiF may be effective at blocking interparticle $\mathrm{Fe}$ diffusion and thereby preventing coarsening. It is also interesting to note that the lattice of the Fe particles in the near-surface region is slightly larger (by $\sim 4 \%$ ) compared with the lattice of the slightly larger particles in the centre as seen in the fast Fourier transform of Fig. 1d. This type of lattice expansion may be attributed to a nano-size effect, as similar expansions have been observed in metal nanoparticles with particle dimensions in a similar range ${ }^{18,19}$. The $\mathrm{Fe}$ crystallites, converted within the same $\mathrm{FeF}_{2}$ particle, tend to be interconnected and aligned in the same crystallographic orientation (Fig. 1d), which has also been observed in previous ex-situ TEM experiments ${ }^{5}$. The distinct morphological feature of $\mathrm{Fe}$ in the near-surface region, that is, forming a chain-like structure (Supplementary Fig. S3), suggests that the phasedecomposition process may occur spontaneously on the surface and propagate into the core.

The reaction within individual particles is surprisingly fast, complete within 3-12 min (listed in Supplementary Table S1), and is equivalent to a discharge rate of $5-20 \mathrm{C}$ in a full battery. Slow kinetics is believed to be intrinsic and insurmountable in conversion electrodes, which would limit their use in real batteries ${ }^{6}$. However, these real-time observations challenge this traditional belief and indicate that rapid cycling rates may be achieved by engineering an electrode with active nanoparticles directly connected to the current collector $^{9}$, rather than the traditional method of preparing heterogeneous nanocomposite electrodes, which often leads to agglomerates of active materials $\mathrm{s}^{3,5,20}$.

A large volume expansion (41-57\%) is observed in the particles during the reaction as the low-density LiF phase is formed, filling in the space around the $\mathrm{Fe}$ particles. As the expected volume expansion is only $21 \%$, based on the crystalline densities these results suggest that the newly formed $\mathrm{Fe}$ and $\mathrm{LiF}$ nanoparticles are not densely packed. Given the low sputtering cross-section of iron 
a

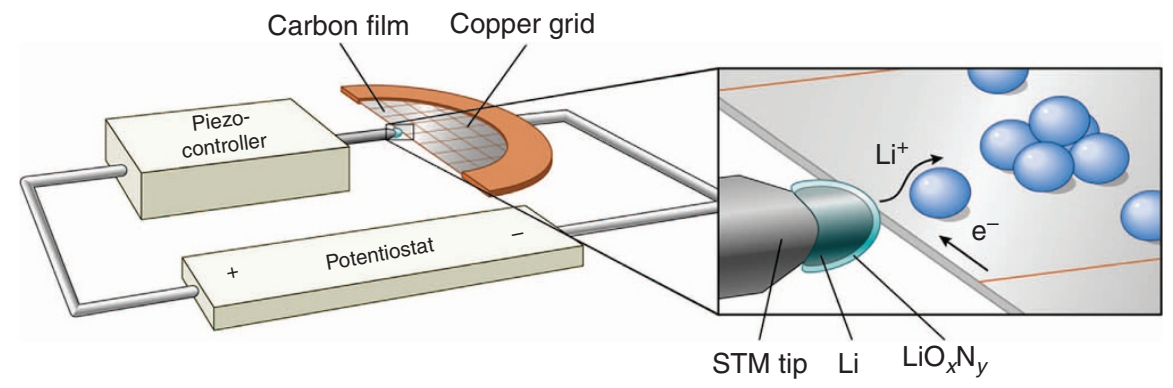

b
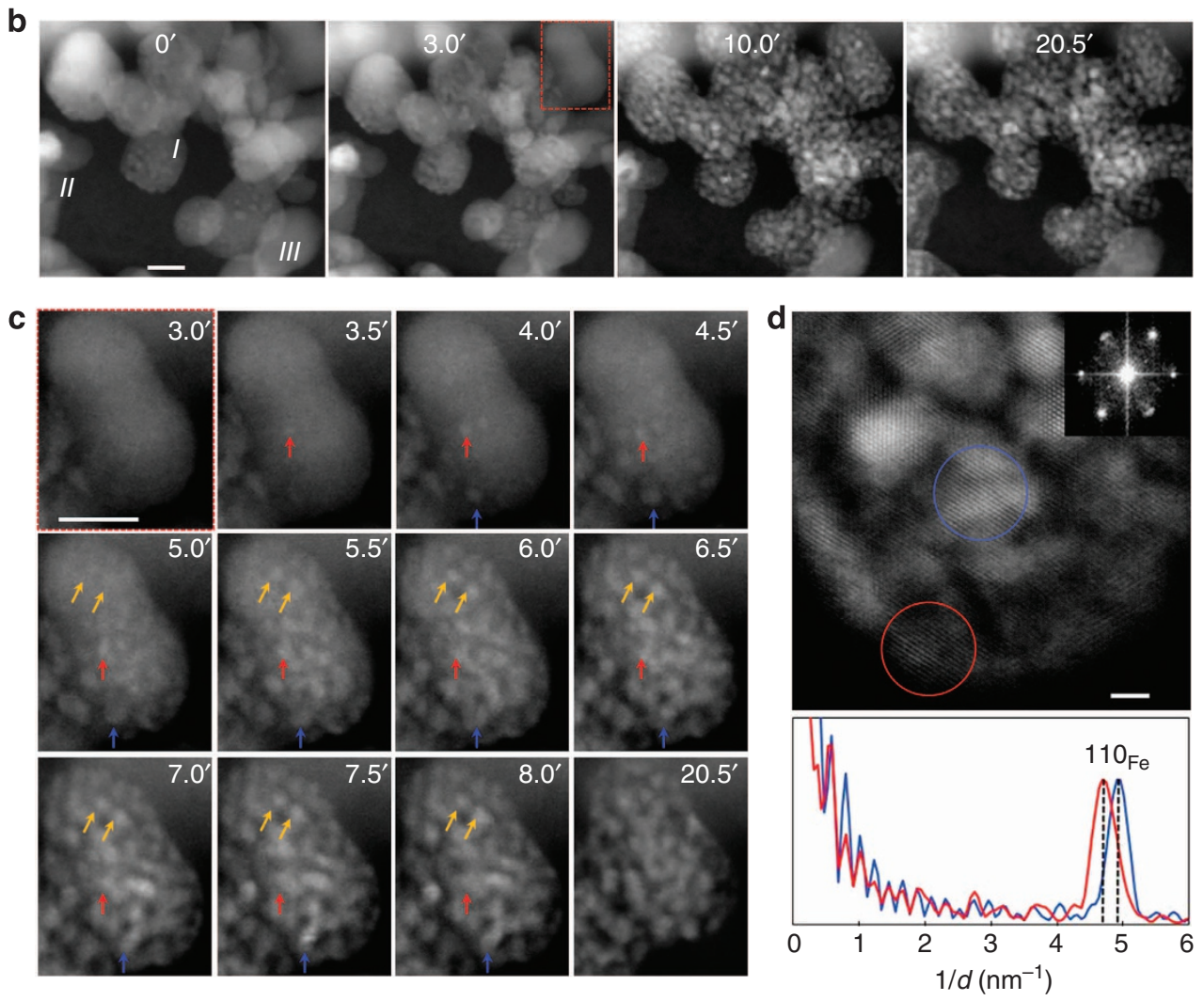

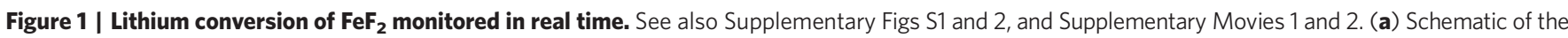
electrochemical cell used for in-situ TEM measurements. (b) Time-lapse images from a collection of particles that react with lithium coming from the lower right. The reaction proceeds immediately in region (I), but is delayed and absent in regions (II) and (III), respectively. Scale bar, $10 \mathrm{~nm}$. (c) Morphological evolution of a single $\mathrm{FeF}_{2}$ nanoparticle, revealing the rapid formation of ultrafine (sub-nm) Fe particles on the surface, followed by gradual formation of larger ones (1-3 nm) within the domain of the original $\mathrm{FeF}_{2}$ particle (arrows used to identify specific Fe particles). Scale bar, $10 \mathrm{~nm}$. (d) Lattice image of Fe particles converted from a single $\mathrm{FeF}_{2}$ particle (top), aligned along the zone $[-1,1,1]$ according to the fast Fourier transform (FFT) pattern (inset), and intensity profiles of the FFT patterns (bottom) from the Fe lattice in the near-surface region (red circle) and central region (blue circle). Scale bar, $2 \mathrm{~nm}$.

at $200 \mathrm{kV}$ ( $\sim 4.1$ barn calculated using the Mott model), little damage is expected to the iron ${ }^{21}$. However, a fraction of the LiF could be destroyed via quick radiolysis. Quasi in-situ measurements were performed with the beam off during the reaction, and with the beam on briefly to record images and spectra of particles immediately before and after lithiation. The TEM images (Supplementary Fig. S4a,b) show that the size and morphology of the Fe particles formed during the quasi in-situ measurements are nearly identical to those formed during the in-situ measurements. The EELS spectra and elemental maps (Supplementary Fig. S4c,d) acquired from the lithiated particles clearly show the presence of LiF and a high spatial correlation with the iron nanoparticles.

Lithium transport in thin films. In-situ measurements were also made on $\mathrm{FeF}_{2}-\mathrm{C}$ film electrodes, composed of a single layer of contiguous $\mathrm{FeF}_{2}$ nanoparticles $(10-20 \mathrm{~nm}$ in diameter) on a 10-nm-thick carbon film (Fig. 2a), which provides the media for tracking the Li transport and a quantitative measure of the propagation of the reaction front. In the ADF images, the bright contrast of the Fe nanoparticles formed on the surface, best seen in the boundary region (Fig. 2b), was used to identify the reaction front, which was observed to propagate rapidly across the film (from lower right to upper left). A plot of the position of the reaction front (distance from the lower right corner, $d$ ) versus time, $t$ (Fig. 2c), was fit to a power law, $t^{n}$ with $n<1$. Rapid $\mathrm{Li}^{+}$surface diffusion clearly has an important role in the advancement of the front. Nevertheless, individual grains undergo a slow reaction process as shown by the time-lapse images in Supplementary Fig. S5. These observations are consistent with those in Fig. 1.

Structural evolution by in-situ ED. A series of ED patterns were recorded, at a frequency of two patterns per second, to monitor the structural evolution of $\mathrm{FeF}_{2}$ particles during the electrochemical reaction. Upon lithiation, the intensity of the diffraction 

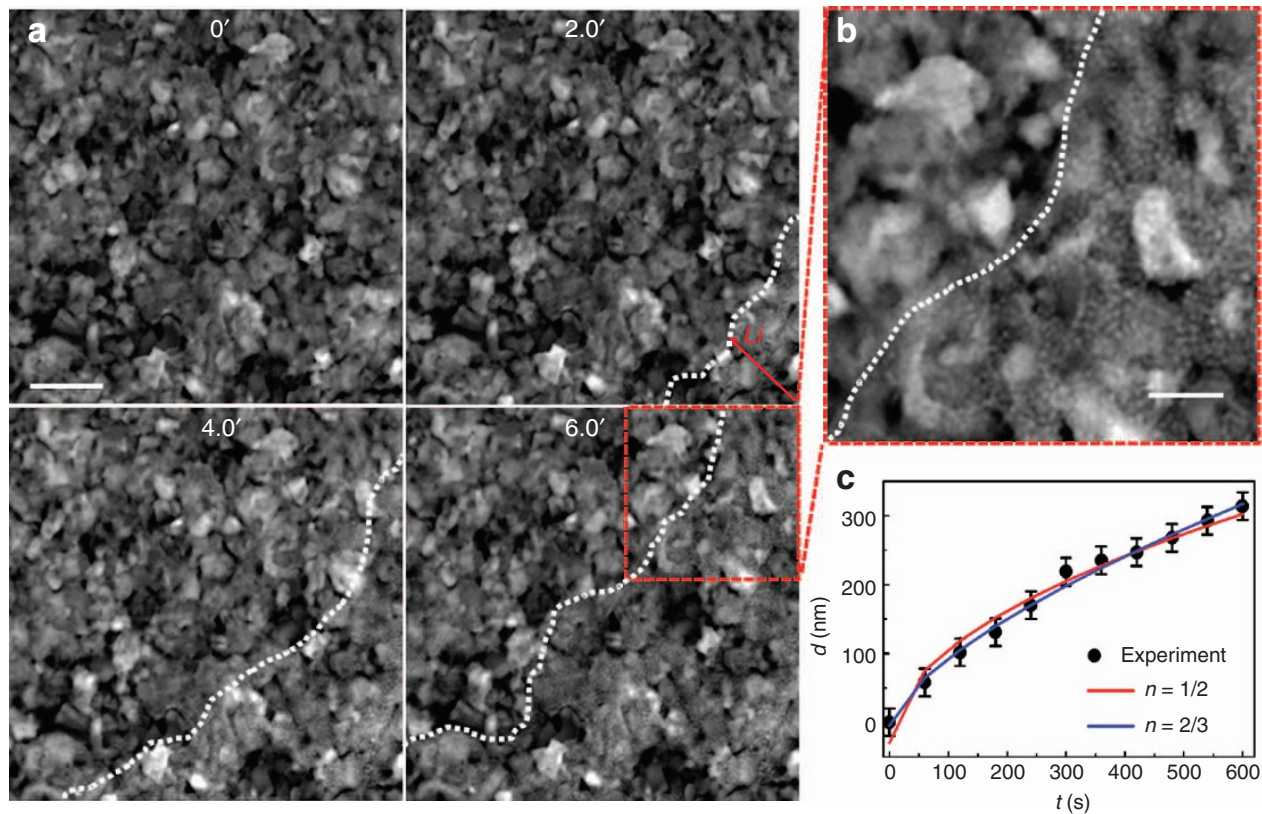

Figure 2 | Lithium conversion in $\mathbf{F e F}_{\mathbf{2}}$ film showing the rapid propagation of the reaction front. See also Supplementary Movie 3. (a) Representative time-lapse images showing the movement of the reaction front across the area, as indicated by the white dashed lines. Scale bar, $50 \mathrm{~nm}$. (b) A magnified view showing the boundary defined with the bright contrast of the newly formed Fe nanoparticles. Scale bar, $20 \mathrm{~nm}$. (c) Distance, $d$, of the reaction front propagation, measured from the lower right corner, as a function of time, $t$, (solid circles), fit with a power law, $\sim t^{n}$, for $n=1 / 2$ (red), $2 / 3$ (blue). The error bars come from the uncertainties in defining the boundaries $(\sim 20 \mathrm{~nm})$.

rings associated with the $\mathrm{FeF}_{2}$ phase decreases along with a corresponding increase in those associated with the Fe phase, as illustrated in Fig. 3. The Bragg rings associated with the Fe phase are broad and diffuse because of the small crystallite size (Supplementary Fig. S6). In the intensity profile (Fig. 3b), there is no noticeable shift in the position of the $\mathrm{FeF}_{2}$ peaks, even at the early stage of the reaction, providing direct evidence of little-tono lithium insertion during the reaction process. The position and intensity of the $\mathrm{Fe}(110)$ peak were estimated through fitting, by which the Fe (110) peak was separated from the neighbouring $\mathrm{FeF}_{2}$ (210) peak (Supplementary Fig. S6c). It should be noted that the contribution from the $\mathrm{Fe}$ (110) peak becomes dominant with lithiation, due to $\sim 60$ times greater Bragg intensity than the $\mathrm{FeF}_{2}$ (210) peak, as predicted by electron structure factor calculations (Supplementary Table S2). The Fe (110) peak initially appears at lower angles (with respect to that of the bulk) and increases in intensity and shifts to higher angles during the electrochemical reaction (Fig. 3c). This shift is consistent with a reaction where small (sub-nanometre) Fe particles, with a slightly larger lattice, nucleate first in the near-surface region (Fig. 1d), followed by the formation of larger (1-3 nm) particles with a 'bulk-like' lattice parameter. This type of lattice expansion was also recently observed in $\mathrm{Fe}$ nanoparticles converted from $\mathrm{Fe}_{2} \mathrm{O}_{3}$ (ref. 22).

Chemical evolution by in-situ EELS. The spectra in the low-loss region, containing the $\mathrm{Li} \mathrm{K}$ - and $\mathrm{Fe}_{2} \mathrm{M}_{23}$-edges were acquired continuously to monitor the chemical evolution upon lithium conversion (Supplementary Movie 5). The representative timelapse spectra shown in Fig. 4a show a gradual fading of the shoulder in the $\mathrm{Fe}_{23}$ peak (at about $55 \mathrm{eV}$ ) and growth of the $\mathrm{Li}$ $\mathrm{K}$-edge (at about $62 \mathrm{eV}$ ) as a result of conversion from $\mathrm{FeF}_{2}$ to $\mathrm{Fe}$ and LiF. Supplementary TEM images and ED patterns were also recorded from the $\mathrm{FeF}_{2}$ particles in the pristine and fully lithiated states (Fig. 4b), providing further information on the phase transition and morphological change. The gradual formation of
LiF during the conversion was clearly detected by in-situ EELS measurements, but the diffraction peaks associated with this phase were hardly detectable (inset of Fig. 4b,c), similar to the observation in Fig. 3. If $\mathrm{LiF}$ is present as a crystalline phase, the strongest scattering ring, $\mathrm{LiF}$ (200), would have an amplitude similar to that of the Fe (200) peak (Supplementary Table S2). The absence of the LiF Bragg rings suggests that this phase may be amorphous when it initially forms, which is consistent with the prediction by molecular dynamics simulations ${ }^{23}$. The disordered LiF phase may be more ionically conductive than crystalline $\mathrm{LiF},{ }^{23}$ but it is not thermodynamically stable at room temperature and crystallizes over time. This may also explain the presence of crystalline $\mathrm{LiF}$ in the fully lithiated $\mathrm{FeF}_{2}$ electrodes examined ex-situ. ${ }^{5}$

\section{Discussion}

In-situ TEM offers a unique glimpse of the nanoscale phase transformations that occur during electrochemical conversion reactions. The processes observed here pose a true challenge to theoretical interpretation, as they take place at length scales too large for direct atomistic simulations, but also too small for a rigorous continuum treatment. Although the converting system is a nanoscale mixture of insulating and metallic phases containing many interfaces, phase stability based on a homogeneous freeenergy description should nevertheless provide some guidance. Figure 5a shows a phase diagram in the ternary Fe-F-Li composition space informed by a large number of first-principles calculations of known and likely structures for this system based on density functional theory (DFT) ${ }^{24}$. The various experimental observations during the conversion reaction are inconsistent with the minimum free-energy path (that is, equilibrium phase sequence dictated by the phase diagram), which predicts that sub-stoichiometric tri-rutile $\mathrm{LiFe}_{2} \mathrm{~F}_{6}$ should form first through a displacement reaction followed by a conversion to $\mathrm{Fe}$ and $\mathrm{LiF}$ (as indicated by the arrow). The predicted voltage curve for this 
a
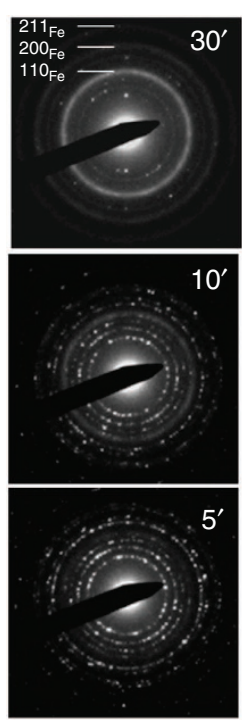

b

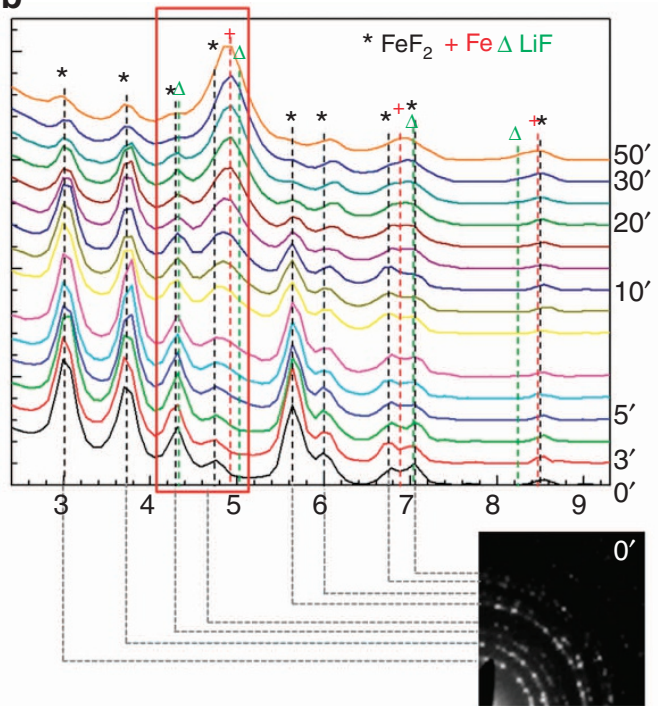

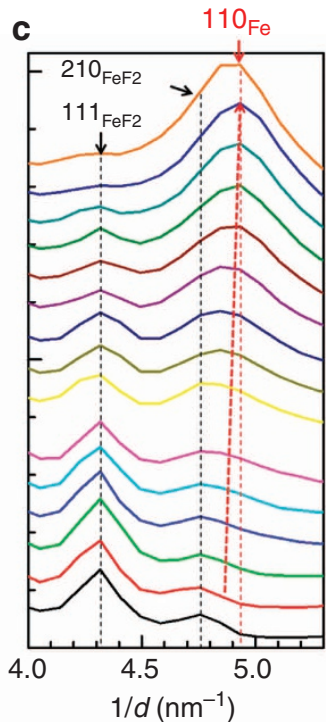

Figure 3 | Structural evolution of $\mathbf{F e F}_{\mathbf{2}}$ nanoparticles by in-situ ED. See also Supplementary Movie 4. (a) Representative ED patterns recorded at 5, 10 and $30 \mathrm{~min}$. (b) Integrated intensity from a selection of ED patterns recorded at different time (labelled on the right side). The correspondence of the peaks with the specific rings in the ED pattern is illustrated for the first pattern (at $0 \mathrm{~min}$ ). (c) A magnified view showing the growth of Fe phase at the expense of the $\mathrm{FeF}_{2}$ phase and the shift of the Fe (110) peak upon lithiation, which is illustrated by a red dashed line (see also Supplementary Fig. S6c for changes in peak position and intensity determined from peak fitting). The expected positions of peaks associated with the three different phases were indicated with different markers: ${ }^{\star} \mathrm{FeF}_{2}$, $+\mathrm{Fe}$ and $\Delta \mathrm{LiF}$.

a

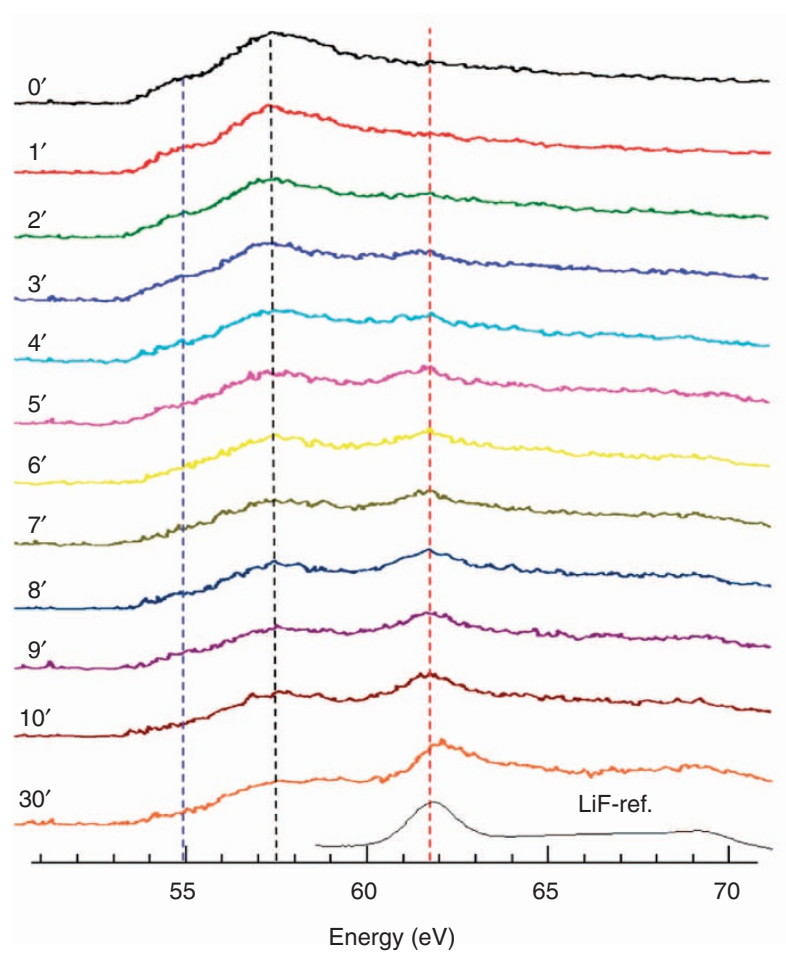

b
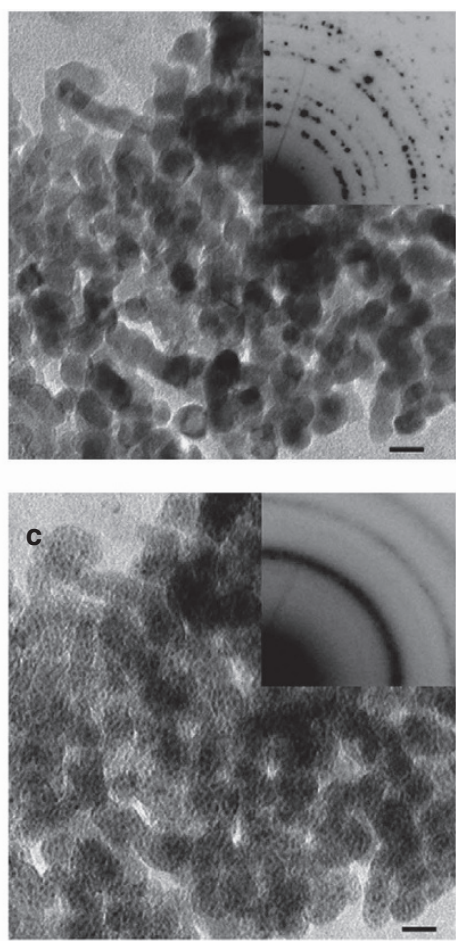

Figure 4 | Chemical evolution of FeF $\mathbf{2}$ nanoparticles by in-situ EELS. See also Supplementary Movie 5. (a) Time-lapse EELS spectra showing the evolution of the chemical states of $\mathrm{Fe}$ and $\mathrm{Li}$, as indicated by the variations in the fine structure of the $\mathrm{Fe}_{23^{-}}$and $\mathrm{Li}$ K-edges during the conversion process. Reference Li K-edge spectrum (LiF-ref.) recorded from LiF compound is shown at the bottom to confirm the formation of LiF, by the characteristic peaks at about 62 and $69 \mathrm{eV}$. (b,c) Bright-field TEM images and ED patterns (inset) of the FeF, nanoparticles in the pristine and fully lithiated states, respectively. Scale bar, $20 \mathrm{~nm}$. 

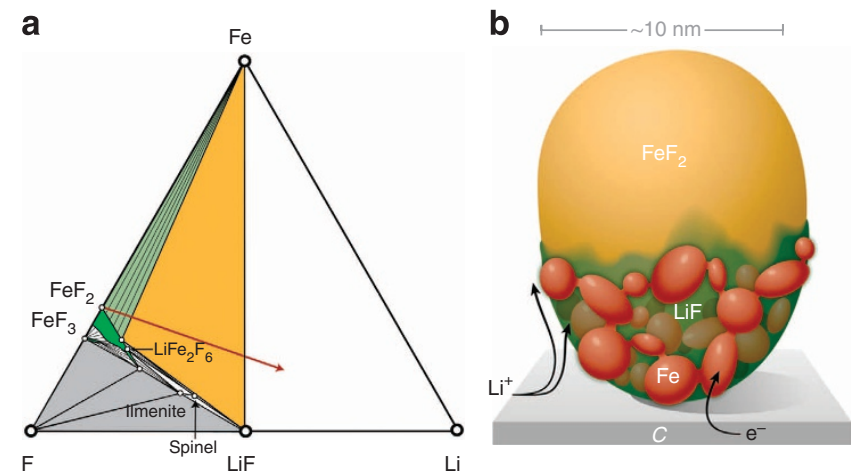

Figure $\mathbf{5}$ | Possible reaction paths in $\mathbf{F e F}_{\mathbf{2}}$. (a) Fe-F-Li phase diagram informed by extensive first-principles calculations of known and candidate structures. (b) Schematic illustration of the propagation of reaction front through a single $\mathrm{FeF}_{2}$ particle via a 'layer-by-layer' process and possible pathway for electron and $\mathrm{Li}^{+}$transport.

equilibrium path (Supplementary Fig. S8) is substantially higher than that measured experimentally ${ }^{5}$. It should be noted that the predicted voltage is a lower bound for the equilibrium path, as we used an energy for pure Fe that accounted for the contribution of surface energies at the nanoscale ${ }^{24}$. In addition, the $\mathrm{Fe}^{3+}$ (expected for $\mathrm{LiFe}_{2} \mathrm{~F}_{6}$ ) was not identified by in-situ EELS measurements (Fig. 4), and the predicted variation of the lattice parameter of rutile $\mathrm{Li}_{x} \mathrm{Fe}_{1-\mathrm{x}} \mathrm{F}_{2}$, of about $12 \%$ (Supplementary Fig. S9), was not detected in the diffraction measurements (Fig. 3). This suggests that the reaction follows one of several possible metastable (non-equilibrium) paths.

One possibility is the direct $\mathrm{Li}$ intercalation into $\mathrm{FeF}_{2}$ until the structure becomes highly supersaturated with $\mathrm{Li}$ upon which it decomposes into LiF and Fe. The voltage predicted under this scenario (Supplementary Fig. S8) is significantly lower than that of the equilibrium path and is also more consistent with the measurements ${ }^{5}$. However, although $\mathrm{Li}$ is predicted to have a very high mobility within rutile $\mathrm{FeF}_{2}$, direct intercalation into $\mathrm{FeF}_{2}$ would lead to energetically undesirable $\mathrm{Fe}^{+1}$ along with a continuous variation in the lattice parameter, which was not observed experimentally. In fact, DFT calculations predict that the rutile crystal structure becomes unstable above a low $\mathrm{Li}$ insertion threshold, relaxing to highly deformed structures that have little in common with the original rutile structure. This suggests that $\mathrm{Li}$ insertion into rutile $\mathrm{FeF}_{2}$ may be accompanied by the amorphization of the host, thereby making it invisible to diffraction probes and explaining the absence of lattice parameter variations with increasing $\mathrm{Li}$ content. The supersaturated amorphous phase can then decompose at a slower rate, requiring the redistribution of more sluggish $\mathrm{Fe}$ and $\mathrm{F}$, not unlike a spinodal decomposition reaction. The resemblance of the meso-structure observed in TEM to spinodal decomposition is striking, as illustrated by the projection of a continuum simulation of spinodal decomposition within an ellipsoidal particle, Supplementary Fig. S10, simulated based on the CahnHilliard equation ${ }^{25}$ with a smoothed boundary method ${ }^{26}$. Nevertheless, this mechanism, which involves the amorphization of the nanoparticles, fails to provide an explanation for the coherent nature of the interconnected Fe network, unless this coherency forms at a later stage, either due to physical rotation of the Fe nanoparticles as they impinge (to eliminate the cost of grain boundary energy) or through a recrystallization mechanism.

An alternative mechanism could be driven by interface processes. Although fluorine mobility within the rutile-like structure is lower than that of $\mathrm{Li}$ or $\mathrm{Fe}$ (Supplementary Fig. S11), the reaction could still be controlled by fluorine redistribution with most of the $\mathrm{F}$ transport occurring along the interfaces that emerge as the two-phase mixture $(\mathrm{LiF}+\mathrm{Fe})$ grows at the expense of $\mathrm{FeF}_{2}$. Such a mechanism would also be consistent with an interconnected network of nanometre-sized Fe particles, as it would essentially be a nanoscale manifestation of a eutectoid reaction. Furthermore, the extraction of $\mathrm{F}$ from rutile $\mathrm{FeF}_{2}$ at its surface could result in the collapse of the body-centred tetragonal Fe sublattice of the rutile structure to form the body-centred cubic crystal structure of pure Fe. This would result in a unique crystallographic orientation of the resultant $\mathrm{Fe}$ relative to the original $\mathrm{FeF}_{2}$ host and could explain the coherency of the Fe network.

Although many questions remain around the true mechanisms behind this conversion reaction, our understanding is distinctly different from what has been speculated about conversion reactions in the absence of these unique in-situ TEM studies. Our observations reveal that $\mathrm{Li}$ ions move quickly along the surface, and then penetrate into the bulk of $\mathrm{FeF}_{2}$ particles at a much slower rate. The reaction path is complex, involving interplay of ion and electron transport dynamics within a nanoscale mixture of multiple phases. A combination of realtime observations with DFT calculations and phase-field simulations seems to suggest a 'layer-by-layer' propagation process in the conversion reaction, as illustrated in Fig. 5b. Diffusion of Li into the bulk is prohibited, but may be allowed locally in the nearsurface region, leading to the supersaturation of a thin layer and immediate spinodal-like phase decomposition. The newly formed Fe nanoparticles and three-phase $\left(\mathrm{Fe} / \mathrm{LiF} / \mathrm{FeF}_{2}\right)$ interfaces provide a pathway for electron and lithium transport into the particle. In contrast to the fast lithiation reaction in intercalation materials that may allow bulk Li diffusion, the conversion reaction is considerably slower, taking a few minutes for an $\sim 10-\mathrm{nm}$ particle, and has strong dependence on the particle size, which may be attributed to the 'layer-by-layer' propagation process.

These results, from a model conversion system $\left(\mathrm{FeF}_{2}\right)$, may shed new light on nanocomposite formation in other metal fluorides and other types of conversion materials (for example, oxides, nitrides and sulphides) ${ }^{27}$. The size and distribution of the nanophases directly impacts the electrochemical performance of these electrodes. The beautiful aspect of the electrochemical conversion process is that it leads to exceptionally fine phase formation, including metals with crystallites $<3 \mathrm{~nm}$, through a self-limited intrinsic reaction. It is worth noting that the electrochemical conversion reaction may also be a useful procedure for preparing unique nanostructured networks, suitable for a variety of applications including catalysis and photoconversion $^{28}$. Although this present study is a distinct step towards understanding the process of electrochemical conversion, additional studies are necessary to better understand how the transport properties of electrons and atomic species influence cycling hysteresis, and the formation and evolution of nanocrystallites. Importantly, the newly developed in-situ cell and methodologies provide an opportunity to track the phase evolution and the formation of intermediate phases (if they occur) during the discharge and charge reactions ${ }^{5,24}$. Future studies will focus on the reconversion (charge) reaction in an attempt to gain new insights into the cycling hysteresis that plague most conversion electrodes.

\section{Methods}

In-situ TEM experiments. An in-situ cell (shown in Fig. 1a) suitable for TEM was developed and used for studying the electrochemical reaction of $\mathrm{FeF}_{2}$ nanoparticles. The samples were directly loaded onto $10 \mathrm{~nm}$ thick amorphous carbon films supported on the copper half-grids. The carbon film has a similar role as the carbon 
black used in general $\mathrm{FeF}_{2}-\mathrm{C}$ electrodes, acting as a support, and also the media for electron and ion transport. A thin layer of lithium metal (as the counter electrode) was coated onto a sharp tungsten tip that was attached to a piezo-driven biasingprobe built into the TEM-scanning tunnelling microscopy sample stage (Nanofactory Instruments $\mathrm{AB}$ ).

All of the components were assembled in an Argon glove box and then transferred into the TEM column using an Ar-filled bag. As illustrated in Fig. 1a, a full battery was built in the TEM by connecting the Li-coated tungsten tip with the carbon support using the piezo controller. The battery was composed of a copper grid (current collector), $\mathrm{FeF}_{2}-\mathrm{C}$ (cathode) and Li metal (anode). A thin passivation layer of $\operatorname{LiN}_{\mathrm{x}} \mathrm{O}_{\mathrm{y}}$ on the surface of the $\mathrm{Li}$ acted as the solid electrolyte ${ }^{29,30}$. The biasing probe was connected to the carbon and the reaction was only initiated by applying a negative bias typically at a value of $2 \mathrm{~V}$ or higher; correspondingly, a low current across the whole electrode was measured, mostly below $60 \mathrm{nA}$. No reaction or current flow occurred before applying the bias or with a lower biasing potential.

In-situ TEM images, ED patterns and EELS spectra were recorded at $200 \mathrm{kV}$ in a JEOL2100F microscope equipped with a Gatan image filter spectrometer. The energy resolution was $\sim 1.1 \mathrm{eV}$, as judged by the full-width at half-maximum of the zero-loss peak. Spectra were recorded either in a scanning TEM (STEM) mode for local analysis, or parallel-beam TEM mode from many particles. ADF-STEM imaging (with $z$-contrast) was employed in this experiment to provide good contrast for the small Fe particles. High-resolution ADF-STEM images were also acquired from the lithiated samples in a dedicated STEM machine (Hitachi 2,700D, with a probe aberration-correction system), to identify the crystallographic orientations of the converted Fe nanoparticles.

During the in-situ TEM measurements, electron beam irradiation was minimized by opening the valve only during the data acquisition. The alignment was performed in a sacrificial region and the measurements were taken on a nearby region that had not been previously exposed to the beam (this procedure was followed in all the in-situ measurements). The total dose was minimized by using the lowest possible magnification and beam current, with some sacrifice in resolution and contrast. Diffraction patterns and EELS spectra were recorded in TEM mode with a spread beam. To examine the irradiation effect on the electrochemical reaction of $\mathrm{FeF}_{2}$ particles, 'quasi in-situ' measurements were also made, with the electron beam off during the reaction, and compared with the insitu measurements.

Fabrication of $\mathbf{F e F}_{\mathbf{2}}$ nanoparticles and thin films. The $\mathrm{FeF}_{2}$ nanoparticles were obtained by a solution process from iron metal and fluorosilicic acid solutions $\left(\mathrm{H}_{2} \mathrm{SiF}_{6}\right)$, as described in ref. 31. The as-prepared $\mathrm{FeF}_{2}$ nanoparticles were 10 $20 \mathrm{~nm}$ in diameter, mostly with well-defined nearly spherical or ellipsoidal shapes. The $\mathrm{FeF}_{2}$ films were fabricated by thermal evaporation in high vacuum, with a base pressure of $10^{-7}$ Torr, at both room temperature and elevated temperature (measured on the substrate). The films were deposited on amorphous 10-nm-thick carbon films. The size of the $\mathrm{FeF}_{2}$ grains varied and was strongly dependent on the temperature of the substrate, from 2 to $3 \mathrm{~nm}$ at $25^{\circ} \mathrm{C}$, to $10-20 \mathrm{~nm}$ at $300^{\circ} \mathrm{C}$ (Fig. 2).

Electron structure factor calculations. The electron structure factor was calculated by

$$
F_{\mathrm{g}}=\sum_{\mathrm{j}} f_{\mathrm{j}}(s) \exp \left(-2 \pi \mathrm{i} \mathrm{g} * r_{\mathrm{j}}\right) \exp \left(-B_{\mathrm{j}} \mathrm{s}^{2}\right)
$$

where $f_{\mathrm{j}}, r_{\mathrm{j}}$ and $B_{\mathrm{j}}$ are the electron atomic scattering amplitude, the atomic position and the Debye-Waller factor, respectively, $g$ is the reciprocal vector, and $s$ is the scattering vector, $s=\mathrm{g} / 2$. In a kinematical approximation (a good approximation for the powder ED of nanoparticles), the intensity of the reflection is

$$
I_{\mathrm{g}} \propto\left(\frac{\gamma F_{\mathrm{g}}}{\pi \Omega}\right)^{2}
$$

where $\gamma$ is the relativistic constant $(\gamma=1.3914$ for an accelerating voltage of $200 \mathrm{kV}$ ), and $\Omega$ is the volume of the unit cell. For powder electron diffraction, the integrated ring intensity is

$$
I_{\mathrm{g}} \propto R_{\mathrm{g}}=M_{\mathrm{g}}\left(\frac{\gamma F_{\mathrm{g}}}{\pi \Omega}\right)^{2} / 2|g|
$$

where $M_{\mathrm{g}}$ is the multiplicity of the reflection $g$. Supplementary Table S2 lists the values for $F_{\mathrm{g}}$ and $R_{\mathrm{g}}$.

First-principles electronic structure calculations. Our first-principles calculations extended those of Doe et al. ${ }^{24}$ to establish phase equilibria in the Li-Fe-F ternary composition space. The calculations were also performed to estimate relative mobilities between $\mathrm{Li}, \mathrm{Fe}$ and $\mathrm{F}$ in the various stable compounds. These first-principles calculations were performed using DFT, with generalized gradient approximation parameterized by Perdew and Wang ${ }^{32}$, as implemented with the Vienna $a b$ initio Simulation Package ${ }^{33}$. Core states were treated with the projectoraugmented wave method ${ }^{34,35}$ using the valence state $3 p^{1} 3 d^{7} 4 s^{1}$ for Fe, $1 s^{1} 2 s^{1} 2 p^{1}$ for $\mathrm{Li}$ and $2 s^{2} 2 p^{5}$ for $\mathrm{F}$. An energy cutoff value of $550 \mathrm{eV}$ was used. Brillioun zone integration was performed on $k$-point meshes that are equivalent or denser than a $6 \times 6 \times 6 k$-point mesh for rutile-unit cell and perovskite unit cell and on an $11 \times 11 \times 11$ mesh for structures based on an face-centered cubic fluorine lattice to obtain a $k$-point convergence of less than $5 \mathrm{meV}$ per atom. All cell parameters and atom positions were allowed to relax fully to achieve energy minimization for each structure. Ferromagnetic, spin-polarized calculations were implemented in all structures containing $\mathrm{Fe}$, as both $\mathrm{FeF}_{2}$ and $\mathrm{FeF}_{3}$ are known to exhibit antiferromagnetic properties below the Neel temperature $\left(T_{\mathrm{N}}\right), 79 \mathrm{~K}$ and $363 \mathrm{~K}$, respectively ${ }^{36,37}$.

To determine phase stability in the Li-Fe-F ternary, we calculated the energies of $\sim 1,360$ different compounds. Of this total, $\sim 890$ of them were rutile-based structures (including trirutile-based structures), $\sim 65$ perovskite-based, $\sim 5$ cryolite-based, $\sim 25$ spinel-based, $\sim 25$ ilmenite-based, $\sim 320$ face-centered cubic fluorine sublattice-based and $\sim 30$ hexagonal close-packed fluorine sublatticebased.

In the construction of the Li-Fe-F equilibrium phase diagram, the Fe reference state was raised by $1 \mathrm{eV}$ to account for several effects ${ }^{24}$. The first is that in generalized gradient approximation calculations, there is an energy discrepancy between the localized $d$-states of the ionic iron in the fluoride and the delocalized states in metallic iron ${ }^{24}$. Another effect to consider is that although the calculations may represent bulk materials, the conversion reactions are only feasible in nanosized particles. Therefore, an adjustment must also be made to account for the difference between bulk and nano materials (estimated for a $3.7 \mathrm{~nm}$ particle) ${ }^{24}$.

The nudged-elastic band method as implemented in Vienna ab initio Simulation Package was used to calculate the migration barriers of Li and Fe. Supercells containing 12 rutile $\mathrm{FeF}_{2}$ unit cells $(2 a \times 2 a \times 3 c)$, where $a$ and $c$ are the primitive unit-cell lattice parameters, were used with a $3 \times 3 \times 2 k$-point grid. All nudgedelastic band calculations were performed at constant volume, after having fully relaxed the initial state of the cell, and a decreased energy cutoff value of $400 \mathrm{eV}$. A total of five images were interpolated between the initial and final states of the migration barrier. The migration barrier of $\mathrm{F}$ was calculated by taking the energy difference between the initial and a final state (thereby giving a lower bound for its migration barrier) within a supercell of four trirutile $\mathrm{LiFe}_{2} \mathrm{~F}_{6}$ unit cells

$(2 a \times 2 a \times 1 c)$ on a $4 \times 4 \times 4 k$-point grid.

The Li migration barrier in $\mathrm{FeF}_{2}$ along the empty tunnels in the (001) direction (Supplementary Fig. S7) is about $90 \mathrm{meV}$, which would indicate high lithium mobility. A slightly more realistic scenario where the valence of $\mathrm{Fe}$ is preserved to be $2+$ is a structure with a dilute concentration of Fe replaced by $\mathrm{Li}\left(\mathrm{Li}_{2} \mathrm{Fe}_{23} \mathrm{~F}_{48}\right)$. In this case, the Li migration barrier in the empty tunnels is $170 \mathrm{meV}$, suggesting high lithium mobility in partially converted structures. Within a structure of the same composition, the Fe migration barrier amounts to $450 \mathrm{meV}$. The $\mathrm{F}$ migration barrier in an even further converted structure, namely trirutile $\left(\mathrm{Li}_{8} \mathrm{Fe}_{16} \mathrm{~F}_{47}\right)$, turns out to be much higher, around $900 \mathrm{meV}$ (Supplementary Fig. S11).

Phase-field simulation. The phase-field method is based on the dynamics driven by the reduction of the total free energy, which leads to the evolution of the order parameter. The order parameter can be either conserved (for example, concentration) or non-conserved (for example, crystallographic orientation). For a simple, conserved system, which can be described by the Cahn-Hilliard equation, the total free energy is approximated by ${ }^{25}$

$$
F=\int\left[f(C)+\frac{\kappa^{2}}{2}|\nabla C|^{2}\right] d \Omega
$$

where $f(C)$ is the bulk free-energy function in terms of the order parameter $C$, and $\kappa$ is the gradient energy coefficient. We employed the smoothed boundary method $^{26}$ to simulate the phase separation confined in the interior of an ellipsoidal particle. The region where phase separation occurs is defined by a domain parameter that specifies the domain in which the evolution equation is solved; $\psi=1$ inside the domain and $\psi=0$ outside the domain. The evolution equation is then reformulated to

$$
\frac{\partial C}{\partial t}=\frac{1}{\psi} \nabla \cdot \psi M \nabla\left[\frac{\partial f}{\partial C}-\kappa^{2} \nabla^{2} C\right] .
$$

To confine the region in which phase separation takes place to an ellipsoidal shell to simulate the conversion of $\mathrm{FeF}_{2}$, the domain parameter profile was calculated by

$$
\psi=\frac{1}{2}\left[\tanh \left(\frac{R_{\mathrm{o}}-r}{\varsigma}\right)-\tanh \left(\frac{R_{\mathrm{i}}-r}{\varsigma}\right)\right]
$$

where $r=\sqrt{\left(x / l_{\mathrm{a}}\right)^{2}+\left(y / l_{\mathrm{b}}\right)^{2}+\left(z / l_{\mathrm{c}}\right)^{2}}$ is the scaled distance to the centre of the ellipsoid particle, located at the origin, $R_{\mathrm{o}}$ is the parameter that determines the size of the ellipsoidal particle, $R_{\mathrm{i}}$ is the parameter that determines the size of the inner surface below which the concentration remains constant at the initial value, and $\varsigma$ is used to control the thickness of the region between $\psi=1$ and $\psi=0$.

Equation (5) is dimensionless and was discretized using the second-order central differencing scheme in space within a domain containing $90 \times 90 \times 128$ Cartesian grid points and a first-order Euler explicit scheme in time. The grid spacing and time step of $\Delta x=1$ and $\Delta t=5 \times 10^{-3}$, respectively, were used. The domain parameter was defined by equation 6 with $l_{\mathrm{a}}=l_{\mathrm{b}}=2, l_{\mathrm{c}}=3$, and $R_{\mathrm{o}}=18$. Four values of $R_{\mathrm{i}}$ were examined $(18,13,9$ and 0$)$ such that the 4 sets of simulations 
represent varying thickness of regions undergoing spinodal decomposition. We set $\varsigma$ to be 0.5 for the domain parameter profile, and the gradient energy coefficient $\kappa$ to be 1.0 to ensure that there were sufficient number of grid points in the interfacial region for numerical accuracy. The initial order parameter for the simulation was set as $C(x, t=0)=0.5+0.005 \eta(x)$, where $\eta(x)$ is given by a random number between -1 and 1 at each grid point, which gives a noise with an amplitude of $1 \%$ of the average concentration.

\section{References}

1. Dunn, B., Kamath, H. \& Tarascon, J. -M. Electrical energy storage for the grid: a battery of choices. Science 334, 928-935 (2011).

2. Poizot, P., Laruelle, S., Grugeon, S., Dupont, L. \& Tarascon, J. -M. Nano-sized transition-metal oxides as negative-electrode materials for lithium-ion batteries. Nature 407, 496-499 (2000).

3. Badway, F., Cosandey, F., Pereira, N. \& Amatucci, G. G. Carbon metal fluoride nanocomposites: structure and electrochemistry of $\mathrm{FeF}_{3}: \mathrm{C}$. J. Electrochem. Soc. 150, A1209-A1218 (2003)

4. Li, H., Balaya, P. \& Maier, J. Li-storage via heterogeneous reaction in selected binary metal fluorides and oxides. J. Electrochem. Soc. 151, A1878-A1885 (2004).

5. Wang, F. et al. Conversion reaction mechanisms in lithium ion batteries: study of the binary metal fluoride electrodes. J. Am. Chem. Soc. 133, 18828-18836 (2011).

6. Balaya, P., Li, H., Kienle, L. \& Maier, J. Fully reversible homogeneous and heterogeneous Li-storage in $\mathrm{RuO} 2$ with high capacity. Adv. Funct. Mater. 13, 621-625 (2003).

7. Bervas, M. et al. Investigation of the lithiation and delithiation conversion mechanisms in bismuth fluoride nanocomposites. J. Electrochem. Soc. 153, A799-A808 (2006).

8. Bruce, P. G., Scrosati, B. \& Tarascon, J. -M. Nanomaterials for rechargeable lithium batteries. Angew. Chem. Int. Ed. 47, 2930-2946 (2008).

9. Taberna, P. L., Mitra, S., Poizot, P., Simon, P. \& Tarascon, J.-M. High rate capabilities $\mathrm{Fe} 3 \mathrm{O} 4$-based $\mathrm{Cu}$ nano-architectured electrodes for lithium-ion battery applications. Nat. Mater. 5, 567-573 (2006).

10. Chao, S. -C. et al. In-situ transmission X-ray microscopy study on working $\mathrm{SnO}$ anode particle of Li-ion batteries. J. Electrochem. Soc. 158, A1335-A1339 (2011).

11. Meirer, F. et al. Three-dimensional imaging of chemical phase transformations at the nanoscale with full-field transmission X-ray microscopy. J. Sync. Rad. 18, 773-781 (2011).

12. Brazier, A. et al. First cross-section observation of an all solid-state lithium-ion 'nanobattery' by transmission electron microscopy. Chem. Mater. 20, 2352-2359 (2008).

13. Yamamoto, K. et al. Dynamic visualization of the electric potential in an all-solid-state rechargeable lithium battery. Angew. Chem. Int. Ed. 49, 4516-4519 (2010).

14. Meng, Y. S. et al. In situ analytical electron microscopy for probing nanoscale electrochemistry. Electrochem. Soc. Interface 20, 49-53 (2011).

15. Huang, J. Y. et al. In situ observation of the electrochemical lithiation of a single $\mathrm{SnO}_{2}$ nanowire electrode. Science 330, 1515-1520 (2010).

16. Wang, C. M. et al. In situ transmission electron microscopy and spectroscopy studies of interfaces in Li ion batteries: challenges and opportunities. J. Mater. Res. 25, 1541-1547 (2010).

17. Wang, F., Malac, M. \& Egerton, R. F. Energy-loss near-edge fine structures of iron nanoparticles. Micron 37, 316-323 (2006).

18. Lu, K. \& Zhao, Y. H. Experimental evidences of lattice distortion in nanocrystalline materials. Nanostr. Mater. 12, 559-562 (1999).

19. Qing, W., Nagase, T., Umakoshi, Y. \& Szpunar, J. A. Relationship between microstrain and lattice parameter change in nanocrystalline materials. Phil. Mag. Lett. 88, 169-179 (2008).

20. Badway, F., Cosandey, F., Pereira, N. \& Amatucci, G. G. Carbon metal fluoride nanocomposites: high-capacity reversible metal fluoride conversion materials as rechargeable positive electrodes for Li batteries. J. Electrochem. Soc. 150, A1318-A1327 (2003).

21. Wang, F. et al. Chemical distribution and bonding state of lithium in intercalated graphite: identification with optimized electron energy-loss spectroscopy. ACS Nano 5, 1190-1197 (2011).

22. Shyam, B. et al. Structural and mechanistic revelations on an iron conversion reaction from pair distribution function analysis. Angew. Chem. Int. Ed. 20, 4636-4639 (2012).

23. Ma, Y. \& Garofalini, S. H. Atomistic insights into the conversion reaction in iron fluoride: a dynamically adaptive force field approach. J. Am. Chem. Soc. 134, 8205-8211 (2012).

24. Doe, R. E., Persson, K. A., Meng, Y. S. \& Ceder, G. First-principles investigation of the Li-Fe-F phase diagram and equilibrium and nonequi- libium conversion reactions of iron fluorides with lithium. Chem. Mater. 20, 5274-5283 (2008).

25. Cahn, J. W. \& Hilliard, J. E. Free energy of a nonuniform system. I. Interfacial energy. J. Chem. Phys. 28, 258-267 (1958).

26. Yu, H.-C., Chen, H.-Y. \& Thornton, K. Extended smoothed boundary method for solving partial differential equations with general boundary conditions on complex boundaries. Model. Simul. Mater. Sci. Eng. 20, 075008 (2012).

27. Cabana, J., Monconduit, L., Larcher, D. \& Palacín, M. R. Beyond intercalationbased Li-ion batteries: state of the art and challenges of electrode materials reacting through conversion reactions. Adv. Mater. 22, E170-E192 (2010).

28. Hu, Y.-S. et al. Electrochemical lithiation synthesis of nanoporous materials with superior catalytic and capacitive activity. Nat. Mater. 5, 713-717 (2006)

29. Islam, M. M. \& Bredow, T. Density functional theory study for the stability and ionic conductivity of $\mathrm{Li}_{2} \mathrm{O}$ surfaces. J. Phys. Chem. C 113, 672-676 (2009)

30. Liu, X. H. et al. Anisotropic swelling and fracture of silicon nanowires during lithiation. Nano Lett. 11, 3312-3318 (2011).

31. Pereira, N., Badway, F., Wartelsky, M., Gunn, S. \& Amatucci, G. G. Iron Oxyfluorides as high-capacity cathode materials for lithium batteries. J. Electrochem. Soc. 156, A407-A416 (2009).

32. Perdew, J. P. \& Wang, Y. Accurate and simple analytic representation of the electron-gas correlation energy. Phys. Rev. B 45, 13244-13249 (1992).

33. Kresse, G. \& Furthmuller, J. Efficient iterative schemes for ab initio totalenergy calculations using a plane-wave basis set. Phys. Rev. B 54, 11169-11186 (1996).

34. Blochl, P. E. Projector augmented-wave method. Phys. Rev. B 50, 17953-17979 (1994).

35. Kresse, G. \& Joubert, D. From ultrasoft pseudopotentials to the projector augmented-wave method. Phys. Rev. B 59, 1758-1775 (1999).

36. Strempfer, J., Rutt, U. \& Bayrakci, S. P. Magnetic properties of transition metal fluorides MF2 ( $\mathrm{M}=\mathrm{Mn}, \mathrm{Fe}, \mathrm{Co}, \mathrm{Ni})$ via high-energy photon diffraction. Phys. Rev. B 69, 014417 (2004).

37. Greneche, J. M. et al. Structural aspects of amorphous iron(III) fluorides. J. Phys. C Solid State Phys. 21, 1351-1361 (1988).

\section{Acknowledgements}

We thank Clare Grey, Gerbrand Ceder, M. Stanley Whittingham and Hong Li for discussions concerning the conversion reaction mechanisms and for critical reading of the manuscript. We thank Dong Su, Lihua Zhang, Eric Stach and Chong-Yong Nam for technical support. This work was supported by the Northeastern Center for Chemical Energy Storage, an Energy Frontier Research Centre funded by the U.S. DOE, BES under award number DE-SC0001294. The research was carried out at the Center for Functional Nanomaterials, Brookhaven National Laboratory, and the work of L.W and Y.Z. (TEM and ED analysis) was supported by the U.S. Department of Energy, Office of Basic Energy Sciences, under contract number DE-AC02-98CH10886. The simulations were performed using computational resources provided under allocation No. TGDMR110007 on Extreme Science and Engineering Discovery Environment (XSEDE), which is supported by National Science Foundation grant number OCI-1053575. Computational resources provided by TERAGRID DMR100093 are also gratefully acknowledged.

\section{Author contributions}

F.W. designed and conducted the experiments, made data analysis and wrote the paper; J.G. initiated the project, assisted with the writing and data interpretation; H.C.Y. and K.T. performed the phase-field simulations; M.C. and A.V.d.V. performed DFT calculations; L.W. and Y.Z. assisted with the data analysis on the HRTEM and ED; N.P. and G.A. synthesized $\mathrm{FeF}_{2}$ nanoparticles; G.A., A.V.d.V. and K.T. provided important insights into the conversion mechanisms; all authors were involved in revising the manuscript.

\section{Additional information}

Supplementary Information accompanies this paper at http://www.nature.com/ naturecommunications

Competing financial interests: The authors declare no competing financial interests.

Reprints and permission information is available online at http://npg.nature.com/ reprintsandpermissions/

How to cite this article: Wang, F. et al. Tracking lithium transport and electrochemical reactions in nanoparticles. Nat. Commun. 3:1201 doi: 10.1038/ncomms2185 (2012). 